\title{
Far-Infrared Spectrum and Hindering Potential of Deuterium Peroxide
}

\author{
ROBERT H. HUNT \\ Department of Physics, The Florida State University, Tallahassee, Florida \\ AND \\ ROBERT A: LEACOCK* \\ The Harrison M. Randall Laboratory of Physics, The University of Michigan, Ann Arbor, Michigan
}

(Received 17 June 1966)

\begin{abstract}
The hindered-rotation motion in the deuterium peroxide molecule is investigated through a study of the far-infrared absorption of the vapor. A $1-\mathrm{m}$ focal-length vacuum grating spectrometer was used to scan the region from 20 to $400 \mathrm{~cm}^{-1}$ with an average resolution of $0.4 \mathrm{~cm}^{-1}$. Seven perpendicular-type hinderedrotation bands characterized by prominent $Q$ branches and unresolved $P$ - and $R$-branch structure are observed in the spectrum. The band centers are located at $1.88,42.3,123.5,136.8,206.7,250.9$, and $302.6 \mathrm{~cm}^{-1}$. From these it is determined that, relative to the ground state, the first five excited hindered-rotation states are at $1.88,208.6,250.9,387.7$, and $511.2 \mathrm{~cm}^{-1}$.

A theory of internal rotation, developed for an earlier application to the far-infrared spectrum of hydrogen peroxide, is applied to the $\mathrm{D}_{2} \mathrm{O}_{2}$ spectrum. In this theory the only internal degree of freedom is the dihedral angle $x$ defining the relative position of the two OD groups. The Hamiltonian is put in the form $H$ (asymmetric top) $+H$ (internal rotation), where the inertial coefficients are functions of the internal angle $x$. A three-parameter hindering potential is assumed, $V(x)=V_{1} \cos x+V_{2} \cos 2 x+V_{3} \cos 3 x$ and the internalrotation wave equation is solved numerically by computer to obtain the potential parameters which reproduce the internal-rotation energy eigenvalues. In the semirigid model adopted, the effective hindering potentials, bond lengths, and bond angles of $\mathrm{H}_{2} \mathrm{O}_{2}$ and $\mathrm{D}_{2} \mathrm{O}_{2}$ differ slightly. The data do not yield a complete set of effective bond lengths and angles for $\mathrm{D}_{2} \mathrm{O}_{2}$, but the product of the OD distance and the sine of the OOD angle is found to be $0.01 \AA$ smaller than its $\mathrm{H}_{2} \mathrm{O}_{2}$ counterpart. As a result, the inertial parameter in the internalrotation wave equation is $2 \%$ larger than is predicted from the $\mathrm{H}_{2} \mathrm{O}_{2}$ data. Using this adjusted inertial parameter, the hindering potential $V(x)=994 \cos x+641 \cos 2 x+55 \cos 3 x$ provides a good fit to the $\mathrm{D}_{2} \mathrm{O}_{2}$ data. For this potential function the $c$ is and trans potential barrier heights are 2470 and $377 \mathrm{~cm}^{-1}$, respectively, and the potential minima are $110.8^{\circ}$ from the cis configuration. These parameter values are similar to the corresponding $\mathrm{H}_{2} \mathrm{O}_{2}$ values of $2460 \mathrm{~cm}^{-1}, 386 \mathrm{~cm}^{-1}$, and $111.5^{\circ}$.
\end{abstract}

\section{INTRODUCTION}

$\mathbf{I}^{\mathrm{N}}$ $\mathrm{N}$ an earlier paper $^{1}$ (hereafter referred to as $\mathrm{I}$ ) a theory of hindered internal rotation was developed for the hydrogen peroxide molecule $\mathrm{H}_{2} \mathrm{O}_{2}$ and its deuterated forms $\mathrm{D}_{2} \mathrm{O}_{2}$ and HOOD. This theory was applied to the analysis of the far-infrared spectrum of $\mathrm{H}_{2} \mathrm{O}_{2}$ vapor and an effective hindering potential function was determined for that molecule. Only a limited study of $\mathrm{D}_{2} \mathrm{O}_{2}$ was possible at that time. Using the microwave data of Massey, Beard, and Jen, ${ }^{2}$ it was shown in I that the first excited hindered-rotation state of $\mathrm{D}_{2} \mathrm{O}_{2}$ was split by about $41 \mathrm{~cm}^{-1}$, and that such a splitting was consistent with that calculated using the $\mathrm{H}_{2} \mathrm{O}_{2}$ hindering potential.

This paper extends the treatment of $\mathrm{D}_{2} \mathrm{O}_{2}$ to include the results of a $0.4-\mathrm{cm}^{-1}$ resolution study of its vapor absorption between 20 and $400 \mathrm{~cm}^{-1}$. Analysis of this spectrum yields the $\mathrm{D}_{2} \mathrm{O}_{2}$ potential parameters, $V($ cis $)=$ $2470 \mathrm{~cm}^{-1}, V(\operatorname{trans})=377 \mathrm{~cm}^{-1}$ with a potential minimum $x_{0}, " 110.8^{\circ}$ from the cis configuration. These

\footnotetext{
* Present address: Department of Physics, Iowa State University, Ames, Ia.

I R. H. Hunt, R. A. Leacock, C. W. Peters, and K. T. Hecht J. Chem. Phys. 42, 1931 (1965)

2 J. T. Massey, C. I. Beard, and C. K. Jen, J. Mol. Spectry. 5,405 (1960).
}

parameter values serve to confirm the spectral analysis of $\mathrm{H}_{2} \mathrm{O}_{2}$ in $\mathrm{I}$ as they differ only slightly from the corresponding $\mathrm{H}_{2} \mathrm{O}_{2}$ values: $V($ cis $)=2460 \mathrm{~cm}^{-1}, V($ trans $)=$ $386 \mathrm{~cm}^{-1}$ and $x_{0}=111.5^{\circ}$. The present data do not uniquely determine the effective bond lengths and angles of $\mathrm{D}_{2} \mathrm{O}_{2}$. However, it is found that the product of the OD distance and the sine of the OOD angle is $0.01 \AA$ smaller than its $\mathrm{H}_{2} \mathrm{O}_{2}$ counterpart.

\section{EXPERIMENTAL}

The spectra were obtained using the 1-m focal-length vacuum grating spectrometer and the aluminum and glass absorption cell described in I. The sample of $\mathrm{D}_{2} \mathrm{O}_{2}$, obtained from Giguère of Laval University, Quebec, Canada, was at the time of its preparation approximately $91 \% \mathrm{D}_{2} \mathrm{O}_{2}$ and $8 \% \mathrm{D}_{2} \mathrm{O}$. Static samples of $\mathrm{D}_{2} \mathrm{O}_{2}$ vapor decomposed too rapidly in the absorption cell to be of use and it was necessary to follow the continuous-flow procedure adopted for $\mathrm{H}_{2} \mathrm{O}_{2} . \mathrm{D}_{2} \mathrm{O}_{2}$ vapor from a liquid sample maintained at about $25^{\circ} \mathrm{C}$ was admitted to the cell at one end and was slowly and continuously withdrawn at the other end by a cold trap and vacuum pump. Pumping speeds sufficient to avoid strong absorption from the decomposition product $\mathrm{D}_{2} \mathrm{O}$ were usually in the range of 1 to $2 \mathrm{ml} / \mathrm{h}$. The $100-\mathrm{ml}$ sample was sufficient to obtain two or more 

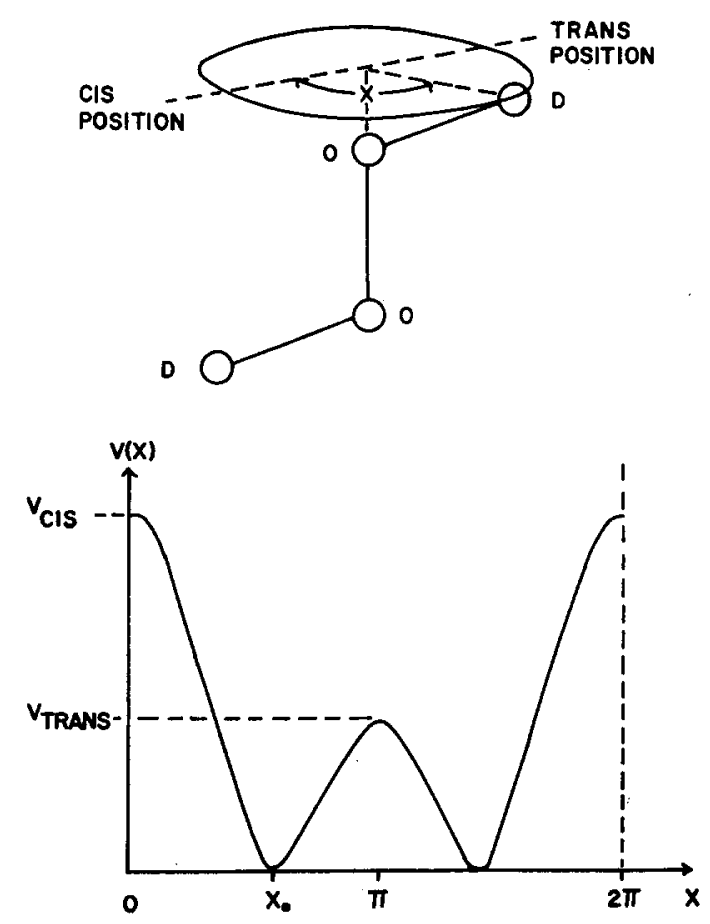

FIG. 1. The structure and hindering potential of $\mathrm{D}_{2} \mathrm{O}_{2}$.

runs over all portions of the $20-$ to $400-\mathrm{cm}^{-1}$ region. The absorption of $\mathrm{D}_{2} \mathrm{O}$ vapor between 20 and $400 \mathrm{~cm}^{-1}$ was also measured as a means of estimating the $\mathrm{D}_{2} \mathrm{O}$ contamination of the $\mathrm{D}_{2} \mathrm{O}_{2}$ spectrum.

Absorption paths in the 0.4-m-long White cell ranged from $1.6 \mathrm{~m}$ between 20 and $300 \mathrm{~cm}^{-1}$ to $3.2 \mathrm{~m}$ between 300 and $400 \mathrm{~cm}^{-1}$. Because the Golay cell detector suffered a loss in sensitivity at the beginning of this work, the average spectral slit width is $0.4 \mathrm{~cm}^{-1}$ as compared to the $0.3 \mathrm{~cm}^{-1}$ reported for $\mathrm{H}_{2} \mathrm{O}_{2}$.

\section{THEORY}

The theory of internal rotation in $\mathrm{D}_{2} \mathrm{O}_{2}$ is given in I. The results are summarized here. Figure 1 shows the structure of $\mathrm{D}_{2} \mathrm{O}_{2}$ and the form of the internal-rotation hindering potential. A semirigid model is adopted in which the only internal degree of freedom is the angle of relative internal rotation $x$. A set of molecular axes is chosen for which the Hamiltonian has the form [Eq. (3.4) in I]:

$$
\begin{aligned}
H=\beta(x) P^{2}+\nu & (x) P_{Z}{ }^{2}+\gamma(x)\left(P_{X}{ }^{2}-P_{Y}{ }^{2}\right) \\
& +\delta(x)\left(P_{Y} P_{Z}+P_{Z} P_{Y}\right)+\alpha(x) p_{x}{ }^{2}+V(x) .
\end{aligned}
$$

$P$ is the total angular momentum with components $P_{X},{ }^{\mathrm{F}} P_{Y}$, and $P_{Z}$ referred to the molecule-fixed axes $X$, $Y, Z . p_{x}$ is the momentum conjugate to the internalrotation angle $x$, and $V(x)$ is the internal-rotation potential-energy function.

The inertial coefficients $\beta(x), \nu(x), \delta(x), \gamma(x)$, and $\alpha(x)$ are functions of the internal angle $x$, the 00 and OD distances, and the OOD angles. The values of the latter three equivalent parameters in $\mathrm{H}_{2} \mathrm{O}_{2}$ as proposed by Redington, Olson, and $\mathrm{Cross}^{3}$ and as adopted in $\mathrm{I}$ are: $\mathrm{OO}$ distance $1.475 \AA$, $\mathrm{OH}$ distance $0.950 \AA$, and $\mathrm{OOH}$ angle $94.8^{\circ}$.

Because these parameters are effective values for the vibrational ground state of $\mathrm{H}_{2} \mathrm{O}_{2}$ and contain implicit mass dependent contributions from vibrationrotation interactions, the corresponding parameters for $\mathrm{D}_{2} \mathrm{O}_{2}$ are slightly different. However, as remarked, the present data are not sufficient to determine them completely. For this reason the data for $\mathrm{D}_{2} \mathrm{O}_{2}$ are first compared with calculations based on the $\mathrm{H}_{2} \mathrm{O}_{2}$ parameters and the latter are then modified as required. Using the $\mathrm{H}_{2} \mathrm{O}_{2}$ bond lengths and angles, the following expansions of the inertial coefficients of $\mathrm{D}_{2} \mathrm{O}_{2}$ are obtained in $\mathrm{cm}^{-1}$ (see Appendix III of I) :

$$
\begin{aligned}
& \beta(x)=0.772+0.00692 \cos x+0.00241 \cos 2 x+\cdots, \\
& \nu(x)=4.598-0.135 \cos x+0.0134 \cos 2 x+\cdots, \\
& \gamma(x)=0.00184+0.0550 \cos x+0.00098 \cos 2 x+\cdots, \\
& \alpha(x)=21.277+0.190 \cos x+0.0960 \cos 2 x+\cdots, \\
& \delta(x)=0.146 \sin ^{3 \frac{3}{2} x+\cdots} .
\end{aligned}
$$

In lowest order, the Hamiltonian separates into that of a rigid symmetric top plus an internal-rotation Hamiltonian $\alpha(x) p_{x}{ }^{2}+V(x)$. The eigenfunctions of the latter Hamiltonian are denoted by $M_{n \tau}(x) . n$ is a principal quantum number ordering the internalrotation energy levels of a given symmetry species $\tau$. The symmetries of $M_{n \tau}$ under the operations $\sigma_{t}$ and $\sigma_{c}$ (reflections in the trans and cis planes, respectively) are given in Table I.

The hindering potential is assumed to have the form $V(x)=V_{1} \cos x+V_{2} \cos 2 x+V_{3} \cos 3 x$. The resulting Mathieu-type internal-rotation wave equation is solved by computer for appropriate values of the input param-

\begin{tabular}{|c|c|c|c|}
\hline \multirow{2}{*}{$\begin{array}{c}K \text { value } \\
\text { (even or odd) }\end{array}$} & \multicolumn{2}{|c|}{$\begin{array}{c}\text { Symmetry } \\
\text { under }\end{array}$} & \multirow{2}{*}{$\begin{array}{c}\tau \text { quantum } \\
\text { number }\end{array}$} \\
\hline & $\sigma_{t}$ & $\sigma_{c}$ & \\
\hline $\mathrm{e}$ & $\boldsymbol{s}$ & $s$ & 1 \\
\hline 0 & $s$ & $a$ & 2 \\
\hline 0 & $a$ & $s$ & 3 \\
\hline $\mathrm{e}$ & $a$ & $a$ & 4 \\
\hline
\end{tabular}
eters $V_{1}, V_{2}$, and $V_{3}$. The latter are chosen so that the calculated internal-rotation energy eigenvalues coincide with those determined from analysis of the far-infrared spectrum. The computer calculation also gives the internal-rotation eigenfunction $M_{n \tau}(x)$ and the matrix

TABLE I. Symmetry of the internal-rotation wavefunctions.

${ }^{3}$ R. L. Redington, W. B. Olson, and P. C. Cross, J. Chem. Phys. 36, 1311 (1962). 

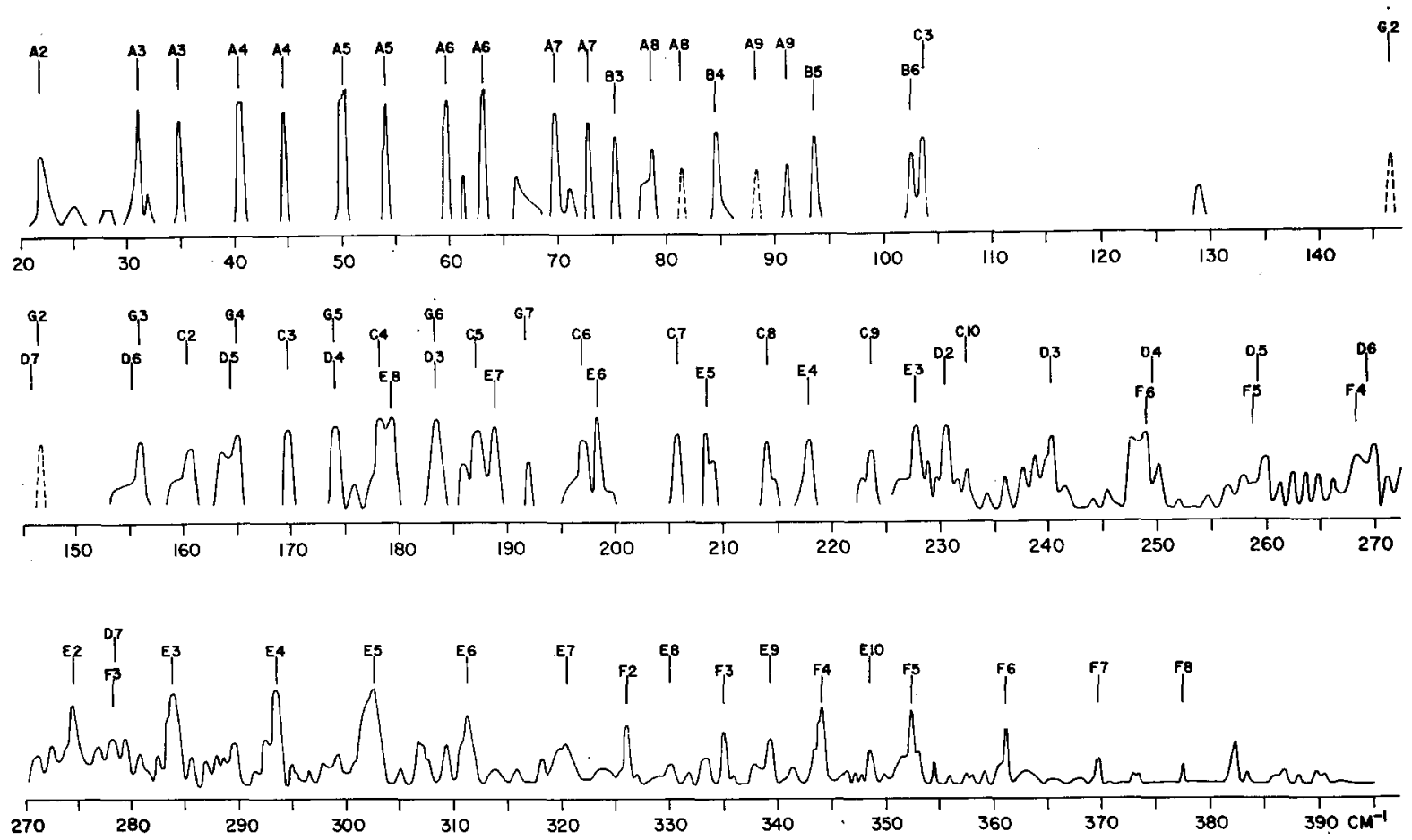

FIG. 2. The absorption spectrum of $\mathrm{D}_{2} \mathrm{O}_{2}$ vapor from 20 to $400 \mathrm{~cm}^{-1}$.

elements of $\cos x, \cos 2 x, \sin ^{3}\left(\frac{1}{2} x\right)$, and $\cos \left(\frac{1}{2} x\right)$ for use in the perturbation-theory calculation.

The full Hamiltonian matrix is then written using the basis functions

$$
\begin{aligned}
\psi_{J\left(K_{ \pm}\right) M n \tau} & =1 \sqrt{2}\left(\psi_{J K M} \pm \psi_{J(-K) M}\right) M_{n \tau} \\
\psi_{J O M}{ }_{n \tau} & =\psi_{J O M} M_{n \tau},
\end{aligned}
$$

where the $\psi_{J K M}$ are the symmetric-top wavefunctions. Since the off-diagonal (in $K$ ) matrix elements of the full Hamiltonian are small, the energy levels of the semirigid model of the molecule can be calculated to an accuracy of several hundredths of a wavenumber using second-order perturbation theory.

The zeroth-order selection rules for hindered-rotation transitions are discussed in $\mathbf{I}$ and are summarized here in Table II.

\section{RESULTS}

The absorption spectrum of $\mathrm{D}_{2} \mathrm{O}_{2}$ vapor between 20 and $400 \mathrm{~cm}^{-1}$ is shown in Fig. 2. In some regions the weaker absorption lines have been omitted because of the difficulty in distinguishing the contribution from $\mathrm{D}_{2} \mathrm{O}$. Dashed portions of the spectrum indicate where moderately strong lines of $\mathrm{D}_{2} \mathrm{O}$ and $\mathrm{D}_{2} \mathrm{O}_{2}$ overlap, rendering the intensity of the latter quite uncertain.

There is no strong evidence for $\mathrm{HOOD}, \mathrm{H}_{2} \mathrm{O}$, or $\mathrm{HDO}$ absorption arising from the sample. Some spectra did show the strongest rotational lines of $\mathrm{H}_{2} \mathrm{O}$ to a slight extent, but these were due to the modest vacuum attainable in the source housing. The spectrum of $\mathrm{D}_{2} \mathrm{O}$, run for comparison purposes, is in good agreement with that of Fuson, Randall, and Dennison. ${ }^{4}$

Table III lists the frequencies of the major absorption lines of $\mathrm{D}_{2} \mathrm{O}_{2}$ vapor measured at the absorption peaks. The estimated error in the measurement of welldefined absorption peaks is $\pm 0.05 \mathrm{~cm}^{-1}$. A comparison of the peak percent absorption for the same path length is also given.

As in the case of $\mathrm{H}_{2} \mathrm{O}_{2}$, the hindered-rotation bands of $\mathrm{D}_{2} \mathrm{O}_{2}$ are perpendicular in character with prominent $Q$ branches (except near the band centers) and unresolved $P$ - and $R$-branch structure. Seven such bands have been identified. They are analyzed using the positions of the $Q$-branch absorption peaks as approximate $Q$-subband heads. The positions of the latter in the $n \tau \rightarrow n^{\prime} \tau^{\prime}$ hindered-rotation band are then fitted

TABLE II. Selection rules for hindered-rotation bands of $\mathrm{D}_{2} \mathrm{O}_{2}$.

\begin{tabular}{cc}
\hline \hline$J=0$ transitions & $\Delta J= \pm 1$ transitions \\
\hline$(K+) \rightarrow(K+) \pm 1$ & $(K+) \rightarrow(K-) \pm 1$ \\
$(K-) \rightarrow(K-) \pm 1$ & $(K-) \rightarrow(K+) \pm 1$ \\
$n \rightarrow n^{\prime}$ & $n \rightarrow n^{\prime}$ \\
$\tau=1 \rightarrow 3$ or $=2 \rightarrow 4$ & $\tau=1 \rightarrow 3$ or $=2 \rightarrow 4$ \\
\hline
\end{tabular}

${ }^{4}$ N. Fuson, H. M. Randall, and D. M. Dennison, Phys. Rev. $56,982(1939)$. 
TABLE III. Frequencies of the principal absorption lines of $\mathrm{D}_{2} \mathrm{O}_{2}$ vapor between 20 and $400 \mathrm{~cm}^{-1}$.

\begin{tabular}{|c|c|c|c|c|c|}
\hline $\begin{array}{l}\text { Frequency } \\
\left(\mathrm{cm}^{-1}\right)\end{array}$ & $\begin{array}{c}\text { Percent } \\
\text { absorption }\end{array}$ & $\underset{\left(\mathrm{cm}^{-1}\right)}{\text { Frequency }}$ & $\begin{array}{l}\text { Percent } \\
\text { absorption }\end{array}$ & $\begin{array}{c}\text { Frequency } \\
\left(\mathrm{cm}^{-1}\right)\end{array}$ & $\begin{array}{c}\text { Percent } \\
\text { absorption }\end{array}$ \\
\hline 21.83 & 35 & 169.68 & 40 & 269.71 & 30 \\
\hline 31.24 & 55 & 173.81 & 40 & 274.83 & 35 \\
\hline 34.97 & 50 & 178.42 & 45 & 278.23 & 20 \\
\hline 40.66 & 55 & 179.30 & 45 & 279.47 & 20 \\
\hline 44,46 & 50 & 183.22 & 45 & 283.89 & 40 \\
\hline 50.24 & 60 & 186.30 & 20 & 293.35 & 40 \\
\hline 53.90 & 55 & 187.52 & 35 & 302.60 & 40 \\
\hline 59.71 & 55 & 188.84 & 40 & 307.02 & 20 \\
\hline 63.32 & 65 & 191.88 & 20 & 309.20 & 20 \\
\hline 69.13 & 50 & 196.47 & 30 & 311.78 & 30 \\
\hline 72.62 & 45 & 198.33 & 45 & 320.6 & 20 \\
\hline 75.27 & 40 & 205.56 & 35 & 325.99 & 25 \\
\hline 78.57 & 35 & 208.0 & 35 & 330.13 & 10 \\
\hline 84.35 & 45 & 214.34 & 30 & 333.5 & 15 \\
\hline 91.31 & 30 & 217.69 & 30 & 334.95 & 25 \\
\hline 93.63 & 40 & 223.55 & 25 & 339.23 & 20 \\
\hline 102.51 & 35 & 227.42 & 35 & 343.60 & 35 \\
\hline 103.52 & 40 & 230.78 & 35 & 348.44 & 15 \\
\hline 129.22 & 15 & 232.19 & 15 & 352.46 & 35 \\
\hline 146.6 & $\cdots$ & 240.23 & 30 & 360.90 & 25 \\
\hline 155.78 & 35 & 248.0 & $\cdots$ & 369.29 & 15 \\
\hline 160.59 & 30 & 250.17 & 20 & 377.43 & 10 \\
\hline 163.8 & 25 & 259.90 & 25 & 382.59 & 20 \\
\hline 165.00 & 35 & 268.75 & 25 & & \\
\hline
\end{tabular}

to the equation

$$
\begin{aligned}
& Q_{K}(0)=\nu_{n^{\prime} \tau^{\prime}} n^{\prime \tau^{\prime}}(K \pm 1)^{2}-v_{n \tau}{ }^{n \tau} K^{2}+E_{n^{\prime} \tau^{\prime}} \\
& -E_{n \tau}-D_{K}\left[(K \pm 1)^{4}-K^{4}\right]
\end{aligned}
$$

using combination relations wherever possible. $D_{K}$ is a distortion coefficient added empirically to include vibration-over-all-rotation interactions. The upper sign applies to ${ }^{R} Q_{K}$ branches, the lower sign to ${ }^{P} Q_{K}$ branches. $E_{n \tau}$ is the energy of the $n \tau$ internal-rotation state and the rotational constant $\nu_{n \tau}{ }^{n r}$ is the expectation value of $\nu(x)$ in that state.

Table IV lists the hindered-rotation band centers obtained and the internal-rotation transitions from which they arise. The errors quoted for the band centers are based on the analyses of the individual bands except for Bands $D$ and $G$. The centers of these bands are more precisely determined from the energy-level positions obtained from the other bands.

Experimental values for $\nu_{n \tau}{ }^{n \tau}$ are given in Table V along with a value for $D_{K}$.

TABLE IV. Observed $\mathrm{D}_{2} \mathrm{O}_{2}$ band centers and band assignments.

\begin{tabular}{ccc}
\hline \hline & $\begin{array}{c}\text { Band center } \\
\left.\text { (in cm } \mathrm{cm}^{-1}\right)\end{array}$ & $\begin{array}{c}\text { Internal-rotation } \\
\text { transition } \\
n \rightarrow n^{\prime} \tau^{\prime}\end{array}$ \\
\hline $\mathrm{A}$ & $1.88 \pm 0.06$ & $01 \rightarrow 03,02 \rightarrow 04$ \\
$B$ & $42.3 \pm 0.2$ & $11 \rightarrow 13,12 \rightarrow 14$ \\
$C$ & $136.8 \pm 0.2$ & $13 \rightarrow 21,14 \rightarrow 22$ \\
$D$ & $206.7 \pm 0.3$ & $03 \rightarrow 11,04 \rightarrow 12$ \\
$E$ & $250.9 \pm 0.1$ & $01 \rightarrow 13,02 \rightarrow 14$ \\
$F$ & $302.6 \pm 0.3$ & $11 \rightarrow 23,12 \rightarrow 24$ \\
$G$ & $123.5 \pm 0.3$ & $21 \rightarrow 23,22 \rightarrow 24$ \\
\hline
\end{tabular}

Figure 3 compares the observed $\mathrm{D}_{2} \mathrm{O}_{2}$ internal-rotation energy-level scheme with that calculated from the bond constants and hindering potential of $\mathrm{H}_{2} \mathrm{O}_{2}$. The letters labeling the internal-rotation transitions correspond to the letters of Fig. 2 and Table IV. Several matrix elements calculated with the $\mathrm{H}_{2} \mathrm{O}_{2}$ potential are given in Table VI. Comparison of Tables V and VI and inspection of Fig. 3 reveals that the effective hindering potentials and bond constants of $\mathrm{H}_{2} \mathrm{O}_{2}$ and $\mathrm{D}_{2} \mathrm{O}_{2}$ differ only slightly.

Absence of experimental values for $\gamma_{n r}{ }^{n \tau}$ and $\beta_{n \tau}{ }^{n \tau}$ prevents a unique determination of the $\mathrm{OO}$ and $\mathrm{OD}$ distances and the OOD angle. However, since $\nu_{01}{ }^{01}$ as calculated from $\mathrm{H}_{2} \mathrm{O}_{2}$ data is $0.094 \mathrm{~cm}^{-1}$ smaller than the experimental value of $4.742 \mathrm{~cm}^{-1}$, it follows that the product of the OD distance and the sine of the OOD angle is $0.936 \pm 0.006 \AA$, i.e., $0.01 \AA$ smaller than its $\mathrm{H}_{2} \mathrm{O}_{2}$ counterpart. This is because $\nu(x)$, in first approximation, varies as the inverse square of the above product and depends only weakly on other functions of the bond constants. This is also true of $\alpha(x)$, the inertial coefficient in the internal-rotation wave equation. As a result, $\alpha_{0}$, the major term in $\alpha(x)$, has an

TABLE V. Experimental values of $\nu_{n+}^{n \tau}$ for $n=0,1,2$.

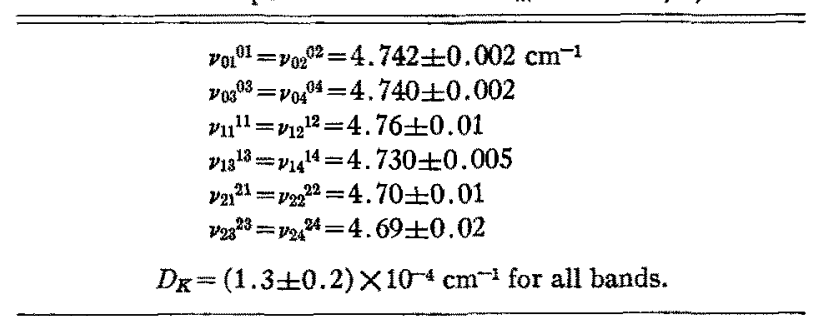




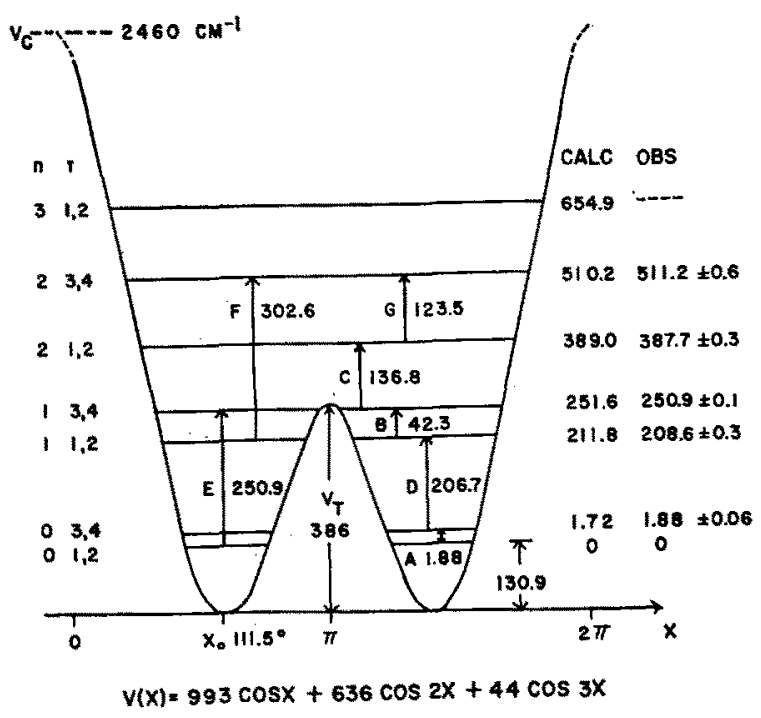

FIG. 3. Comparison of the observed $\mathrm{D}_{2} \mathrm{O}_{2}$ internal-rotation energy levels with those calculated using the $\mathrm{H}_{2} \mathrm{O}_{2}$ hindering potential function and bond parameters.

effective value in $\mathrm{D}_{2} \mathrm{O}_{2}$ of $21.7 \pm 0.1 \mathrm{~cm}^{-1}$ as compared with the value of $21.3 \mathrm{~cm}^{-1}$ calculated from $\mathrm{H}_{2} \mathrm{O}_{2}$ data. The best fit to the experimental level positions based on this increased value of $\alpha_{0}$ is then obtained with the $\mathrm{D}_{2} \mathrm{O}_{2}$ hindering potential function $V(x)=994 \cos x+$ $641 \cos 2 x+55 \cos 3 x$. This potential is characterized by

$$
\begin{aligned}
V(\text { trans }) & =377 \mathrm{~cm}^{-1}, \\
V(\text { cis }) & =2470 \mathrm{~cm}^{-1}, \\
x_{0} & =110.8^{\circ} .
\end{aligned}
$$

The first five eigenvalues above the ground state calculated with this potential are 1.86, 208.7, 251.0, 387.1, and $510.7 \mathrm{~cm}^{-1}$ in good agreement with the experimental values. The ground state is $130.7 \mathrm{~cm}^{-1}$ above the potential minimum.

\section{DISCUSSION}

The $1.88-\mathrm{cm}^{-1}$ band, whose $Q$ branches are labeled $\mathrm{A}$ in Fig. 2, is well fitted by a perpendicular band analysis and it is from this band that $D_{K}$ is determined. Table VII shows the observed $Q$-branch frequencies for this

TABLE VI. Some computed matrix elements of $\mathrm{D}_{2} \mathrm{O}_{2}$ based on $\mathrm{H}_{2} \mathrm{O}_{2}$ parameters.

\begin{tabular}{cccc}
\hline$n \tau$ & $\begin{array}{c}\beta_{n t}{ }^{n \tau} \\
\left(\mathrm{cm}^{-1}\right)\end{array}$ & $v_{n s}{ }^{n \tau}$ & $\gamma_{n \tau}{ }^{n+}$ \\
\hline 01,02 & 0.7681 & 4.648 & -0.0220 \\
03,04 & 0.7681 & 4.646 & -0.0213 \\
11,12 & 0.7681 & 4.685 & -0.0330 \\
13,14 & 0.7684 & 4.658 & -0.0242 \\
21,22 & 0.7686 & 4.670 & -0.0274 \\
23,24 & 0.7690 & 4.650 & -0.0205 \\
\hline
\end{tabular}

Table VII. Calculated and observed Q-branch frequencies of the $1.88-\mathrm{cm}^{-1}$ band.

\begin{tabular}{cccccc}
\hline & \multicolumn{2}{c}{$n \tau=01 \rightarrow 03,02 \rightarrow 04$} & & \multicolumn{2}{c}{$n \tau=03 \rightarrow 01,04 \rightarrow 02$} \\
\cline { 2 - 3 } \cline { 5 - 6 } & Calc & Obs & & Calc & $\begin{array}{c}\text { Obs } \\
\left(\mathrm{cm}^{-1}\right)\end{array}$ \\
\hline $2 \rightarrow 3$ & 25.56 & $\cdots$ & & 21.83 & 21.83 \\
$3 \rightarrow 4$ & 35.02 & 34.97 & & 31.31 & 31.24 \\
$4 \rightarrow 5$ & 44.46 & 44.46 & & 40.78 & 40.66 \\
$5 \rightarrow 6$ & 53.88 & 53.90 & & 50.24 & 50.24 \\
$6 \rightarrow 7$ & 63.28 & 63.32 & & 59.69 & 59.71 \\
$7 \rightarrow 8$ & 72.66 & 72.62 & & 69.13 & 69.13 \\
$8 \rightarrow 9$ & 82.01 & $\mathrm{a}$ & & 78.54 & 78.57 \\
$9 \rightarrow 10$ & 91.33 & 91.31 & & 87.93 & $\mathrm{a}$ \\
\hline \hline
\end{tabular}

Largely obscured by $\mathrm{D}_{2} \mathrm{O}$.

band and the same frequencies calculated using the constants of Tables IV and V. Table VIII gives similar comparisons for the remaining bands. The $Q$ branches of these bands are identified in Fig. 2 by the same letter used to label the bands in Fig. 3 and by a number corresponding to the initial $K$ value involved.

A relatively large number of absorption lines have been assigned to $Q$ branches of the $250.9-\mathrm{cm}^{-1}$ band. Assuming these assignments are correct, it is not understood why ${ }^{R} Q_{8}$ appears much weaker than ${ }^{R} Q_{9} .{ }^{R} Q_{11}$ is the first $Q$ branch which might be affected by a resonance arising from the Hamiltonian term

$$
\gamma(x)\left(P_{X}^{2}-P_{Y}^{2}\right)
$$

No ${ }^{P} Q$ branches of the $302.6 \mathrm{~cm}^{-1}$ band, except possibly ${ }^{P} Q_{4}$, can be identified with great certainty. They appear to be overlapped by the diffuse ${ }^{R} Q_{K}$ branches of the much stronger $206.7-\mathrm{cm}^{-1}$ band. Also, ${ }^{R} Q_{0},{ }^{P} Q_{1}$, etc., of the $250.9-\mathrm{cm}^{-1}$ band must contribute a considerable background of absorption in this region.

Series I of the microwave spectrum of Massey, Beard, and $\mathrm{Jen}^{2}$ was ascribed in $I$ to the transitions $J,(K \pm), n, \tau=J,(4 \pm), 1,4 \rightarrow J,(5 \pm), 1,2$. This assignment is confirmed here by the observation of several other $Q$ branches of this band which is centered at $42.3 \mathrm{~cm}^{-1}$. In I this band was placed at $41.1 \mathrm{~cm}^{-1}$. This number was based on values of $\nu_{n \tau}{ }^{n \tau}$ calculated from $\mathrm{H}_{2} \mathrm{O}_{2}$ data which, as a comparison of Tables $\mathrm{V}$ and VI shows, are somewhat smaller than the experimental values. The head of this microwave series occurs at $1.28 \mathrm{~cm}^{-1}$. The value calculated from the present data is $0.97 \mathrm{~cm}^{-1}$. The errors quoted for the band constants are sufficient to encompass this difference, part of which may be due to the approximation of taking the observed absorption peaks as the $Q$-subband heads.

The $136.8-\mathrm{cm}^{-1}$ band is well behaved. At this low frequency the asymmetry in the intensity distribution is quite pronounced.

The bands discussed above place the transition $n \tau=21 \rightarrow 23$ at $123.5 \pm 0.3 \mathrm{~cm}^{-1}$, and the transition 
TABLE VIII. Calculated and observed $Q$-branch frequencies for six hindered-rotation bands.

\begin{tabular}{|c|c|c|c|c|c|c|c|c|c|c|c|c|}
\hline \multirow{2}{*}{$\stackrel{Q}{\text { branch }}$} & \multicolumn{2}{|c|}{250.9 band $(E)$} & \multicolumn{2}{|c|}{136.8 band $(C)$} & \multicolumn{2}{|c|}{42.3 band $(B)$} & \multicolumn{2}{|c|}{302.6 band $(F)$} & \multicolumn{2}{|c|}{123.5 band $(G)$} & \multicolumn{2}{|c|}{206.7 band $(D)$} \\
\hline & Calc & Obs & Calc & Obs & Calc & Obs & Calc & Obs & $\mathrm{Calc}^{\circ}$ & Obs & Calc & Obs \\
\hline${ }^{R} Q_{10}$ & 348.46 & 348.44 & 231.93 & $232.19 \mathrm{~cm}^{-1}$ & & & & & & & & \\
\hline${ }^{R} Q_{9}$ & 339.33 & 339.23 & 223.21 & 223.55 & & & & & & & & \\
\hline${ }^{R} Q_{8}$ & 330.24 & 330.13 & 214.46 & 214.34 & & & 377.55 & 377.43 & 200.84 & $\cdots$ & & \\
\hline${ }^{R} Q_{7}$ & 321.04 & 320.6 & 205.60 & 205.56 & 111.58 & a & 369.30 & 369.29 & 192.01 & 191.88 & 278.86 & 279.47 \\
\hline${ }^{R} Q_{6}$ & 311.82 & 311.78 & 196.68 & 196.47 & 102.57 & 102.51 & 360.91 & 360.90 & 183.12 & $183.22^{\mathrm{d}}$ & 269.16 & 269.71 \\
\hline${ }^{R} Q_{5}$ & 302.55 & 302.60 & 187.67 & 187.52 & 93.50 & 93.63 & 352.35 & 352.46 & 174.14 & $173.81^{\mathrm{d}}$ & 259.47 & 259.90 \\
\hline${ }^{R} Q_{4}$ & 293.23 & 293.35 & 178.57 & 178.42 & 84.34 & 84.35 & 343.64 & 343.60 & 165.09 & $165.00^{\mathrm{d}}$ & 249.87 & 250.17 \\
\hline${ }^{R} Q_{3}$ & 283.88 & 283.89 & 169.41 & $169.68^{\mathrm{d}}$ & 75.12 & 75.27 & 334.78 & 334.95 & 155.97 & $155.78^{\mathrm{d}}$ & 240.18 & 240.23 \\
\hline${ }^{R} Q_{2}$ & 274.49 & 274.83 & 160.17 & 160.59 & 65.82 & $\cdots$ & 325.76 & 325.99 & 146.77 & $146.6^{\mathrm{d}}$ & 230.57 & 230.78 \\
\hline${ }^{P} Q_{3}$ & 227.15 & 227.42 & 113.04 & a & & & 278.53 & 278.23 & 99.84 & $\cdots$ & 183.09 & $183.22^{\mathrm{d}}$ \\
\hline${ }^{P} Q_{4}$ & 217.62 & 217.69 & 104.44 & 103.52 & & & 268.67 & 268.75 & 90.28 & $\ldots$ & 173.72 & $173.81^{\mathrm{d}}$ \\
\hline${ }^{P} Q_{5}$ & 208.08 & 208.0 & 93.80 & $93.63^{\mathrm{d}}$ & -0.97 & $-1.28^{b}$ & 258.70 & $\cdots$ & & & 164.41 & $165.00^{\mathrm{d}}$ \\
\hline${ }^{P} Q_{6}$ & 198.53 & 198.33 & & & & & 248.58 & $\ldots$ & & & 155.15 & $155.78^{d}$ \\
\hline${ }^{P} Q_{7}$ & 188.94 & 188.84 & & & & & & & & & 145.94 & $146.6^{\mathrm{d}}$ \\
\hline${ }^{P} Q_{8}$ & 179.40 & 179.30 & & & & & & & & & & \\
\hline${ }^{P} Q_{9}$ & 169.84 & $169.68^{\mathrm{d}}$ & & & & & & & & & & \\
\hline
\end{tabular}

- Presence uncertain, $\mathrm{D}_{2} \mathrm{O}$ strong here.

${ }^{b}$ Microwave value of Massey, Beard, and Jen.2 
$n \tau=03 \rightarrow 11$ at $206.7 \pm 0.3 \mathrm{~cm}^{-1}$. With the exception of ${ }^{R} Q_{2}$, and possibly ${ }^{R} Q_{3}$, the absorptions attributed in Table VIII to the ${ }^{R} Q_{K}$ branches of the 206.7 band appear quite diffuse. Such diffuseness was characteristic of both the higher ${ }^{R} Q_{K}$ and ${ }^{P} Q_{K}$ branches of the analogous band in $\mathrm{H}_{2} \mathrm{O}_{2}$. If this is true for the ${ }^{P} \mathrm{Q}_{K}$ branches here, then the absorption lines at 165.0, 155.8, and $146.6 \mathrm{~cm}^{-1}$ are due largely to the $123.5-\mathrm{cm}^{-1}$ band. As Table VIII shows, a good fit to this band is obtained if the values $\nu_{21}{ }^{21}=4.71 \mathrm{~cm}^{-1}$ and $\nu_{23}{ }^{23}=4.68 \mathrm{~cm}^{-1}$ are adopted. However, if $\nu_{11}{ }^{11}$ is adjusted to $4.77 \mathrm{~cm}^{-1}$, the three lines in question also fit the $206.7-\mathrm{cm}^{-1}$ band reasonably well. Since such adjustments of the rotational constants are within their experimental errors, it is uncertain which band is the major contributor to these lines and it is also likely that these two bands combine to produce the absorption lines at 173.8 and $183.2 \mathrm{~cm}^{-1}$. The line at $191.9 \mathrm{~cm}^{-1}$ assigned to ${ }^{R} Q_{7}$ of the $123.5-\mathrm{cm}^{-1}$ band is not considered to be decisive in the above argument.

Tables VII and VIII show that the agreement between observed and calculated $Q$-branch frequencies is generally poorest for the ${ }^{R} Q_{2}$ and ${ }^{P} Q_{3}$ branches and that, in some cases, these $Q$ branches cannot be identified in the spectrum. In the present notation, these $Q$ branches arise from the transitions $J, K+=J, 2+\leftrightarrow$
$J, 3+$ and $J, K-=J, 2-\leftrightarrow J, 3-$. Calculations using the matrix elements of Table VI show that the first of these transitions is generally strongly $J$ dependent, while the latter is moderately so. Because of unknown vibration-rotation interactions it is difficult to predict which of these $Q$ branches should be observed. However, the following transitions should be spread over several wavenumbers since they all have quartic $J$ coefficients calculated to be greater than $9 \times 10^{-6} \mathrm{~cm}^{-1}$ : All of the $J, 2+\longrightarrow J, 3+$ transitions except those of the 206.7$\mathrm{cm}^{-1}$ band, and all of the $J, 3+\rightarrow J, 2+$ transitions except those of the 123.5 and $302.6-\mathrm{cm}^{-1}$ bands. Thus, in those cases where ${ }^{P} Q_{3}$ and ${ }^{R} Q_{2}$ branches are observed, the contributing component, with the above exceptions, must be largely the $J, 2-\leftrightarrow J, 3$-transitions. This explains why some of these $Q$ branches appear to be of lower intensity than the neighboring ${ }^{R} Q_{3}$ or ${ }^{P} Q_{4}$ branches.

In $\mathrm{H}_{2} \mathrm{O}_{2}$ several $Q$ branches involving $K=1$ were accidentally quite narrow. In $\mathrm{D}_{2} \mathrm{O}_{2}$ the constants are such that this does not occur.

\section{ACKNOWLEDGMENTS}

The authors would like to thank Professor Karl T. Hecht for valuable discussions and Professor Paul A. Giguère for the preparation of the $\mathrm{D}_{2} \mathrm{O}_{2}$ sample. 